16 RESEARCH ITEMS

Prehistoric Finds from

Mr. J. Desmonil LLAR, curator of the Museum at Livingstge tots published two papers (Trans. Roy. Soc. So Africa, 31, Pt. 1) as a result of his visit to Abtosminia on war service. One article deals with a "Fauresmith" site about sixteen miles from Goddar on the edge of the Gondar-Debat plateau. Hete the material used was a fine-grained basalt, and a number of typical hand-axes, cleavers, sideserapers, etc., were collected, as well as several stone balls, which may have been used as 'bolas' stones. These latter are not uncommon in the earlier Stone Age industries of Africa. It is interesting to have found these "Fauresmith" implements so far north as Gondar. The second article deals with several rock-shelter sites near Yavello, a village in the GallaSidama province about five hundred miles south of Addis Ababa and seventy miles from the AbyssiniaKenya border. The finds include Stillbay points, pigmy tools, scrapers of various kinds, flakes, ete. Some of the rock-shelters had been painted, the drawings being very conventionalized. Prof. $\mathrm{H}$. Breuil has already noted two styles of rock-shelter painting in Abyssinia, one more naturalistic, the other very conventionalized. Mr. Clark's rock-shelter paintings would seem to belong to Breuil's latter series. Breuil also collected implements from the rock-shelters he investigated and has suggested that the industry as a whole recalls that of the Magosian culture. The industries from Yavello judging from the drawings, could equally belong the same culture.

Alloxan Diabetes in th Rabbit

IT was shoy shaw Dunn and his collaborators in 1943 that pjedtion of alloxan into animals produced a selectiv focrosis of the $\beta$ cells of the islets of Langerhans. The animals exhibited marked hypoglycæmia duM the first few hours (due to liberation of insulin from the necrotic $\beta$ cells) and afterwards hyperglycæmia until death, which usually occurred within a day or two. E. Duffy (J. Path. Bact., 57, 199; 1946) has succeeded in tiding rabbits over the initial acute and (hitherto fatal) stage by appropriate treatment with glucose or insulin. Such animals survived for many months and exhibited classical symptoms of diabetes mellitus-hyperglycæmia, glycosuria, polyuria, polyphagia. Ketonuria was not present on ordinary diets, though, surprisingly, it could be induced by feeding excess of glucose but not by high fat diets. Post-mortem, the only findings were degeneration and reduction in number of the $\beta$ cells. It is therefore proved that the classical picture of diabetes can be produced by selective damage to the $\beta$ cells of the pancreatic islets.

6 Accelerated Storage Tests to Assess the Quality of Dried Whole-Egg Powder

EGGS in pew 1 ed form have become a well-known commodity (exing the war years, and the extent to which th commodity will compete with other egg profuots during normal times will depend largely on how well its quality can be maintained not only during production but also in handling and storage. Fluorescence appears to be related to protein decom. position in foods, which show more decomposition as spoilage proceeds; and it has been found that the addition of sucrose to egg powder, prior to drying, is effective in delaying fluorescent development at $118^{\circ}$ F. R. L. Hay and J. A. Pearce (Canad. J. Res.,
F, 24, 168; 1946) now show that 33 per cent sucrose in the egg powders has a marked effect in retarding decrease in quality in egg powder as assessed by all quality tests used. Loss in quality is less for sugar. egg powders prepared with granulated sugar than for those prepared with sucrose syrup; and in addition, powder made from fresh shell eggs is more desirable than powder prepared from frozen melange. Present indications are that this sugar-egg powder will find a ready peace-time market for baking and other trade purposes.

\section{Seed Germination and}

J. A. HonIf (Fenetica, 23, 1; 1944) has shown that the ofds f Nicotiana Tabaccum from Sumatra in gen require light for germination, but those frof J $y^{2}$ are light-indifferent. Pure lines are lightind ferent or light-needing. Hybrids between these lines show reciprocal differences when stored in the presence of lime, but the differences largely disappear when storage is done without lime. N. texana also contains strains which have light-sensitive seeds.

\section{Stem Rot of Outfoor Thatoes $\mathrm{Kleb.}$ ) is a poduent cause of loss in outdoor tomato} crops and haky persist from year to year with varying sevitity, on regularly cropped holdings. C. J. Hicl. han (J. Pom. and Hort. Sci., 22, $69 ; 1946)$ has investigated sources of infection in the Evesham area, following the observation that the disease occurred on widely separated holdings supplied with plants from a particular nursery where it had been identified. By mixing seeds from diseased fruits with healthy seeds, samples containing 2 per cent and 70 per cent infected seeds were obtained. Plants raised from these seeds were grown on separate plots, and though no signs of disease were apparent at planting time, a quarter of those from the 2 per cent sample and a third of those from the 70 per cent sample eventually succumbed. Plants from healthy seed became diseased when propagated at nurseries having a previous history of infection, whereas no losses occurred where there was no such history and hygiene was good. Plants in the field were not prone to infection from the soil, even when diseased plants had been ploughed in the previous year. Plants staked with old canes, however, became 38 per cent infected, whereas new canes, or old canes sterilized with formaldehyde, produced no infection. It is concluded that the most probable causes of the disease are spores in propagating soil and spores which have overwintered in cracks of old canes.

\section{Mitotic Activity in the Moure \\ W. S. BuLl ougril. Trans. Roy. Soc., B, 23A,} $435 ; 1946$ has hade an extensive analysis of mitosis in the adfunfouse. The reproductive system, the urinaryfystem, the alimentary canal, various exocridt rand endocrine glands and other organs of the female mouse all exhibit increased mitotic activity in response to cestrogenic hormones. In the ovary the cells are highly resistant to the mitosis-stimulating effect of the œstrogen. The evidence shows the presence of mitosis inhibitors which may be classified as (1) the cell inertia, (2) mitosis depressors. The latter show their effect by taking control immediately after an excess of mitotic activity has been stimulated by œstrogen. Therefore in the presence of a stimulant and the depressant, mitosis proceeds in waves. The proliferating cells of the intestinal lymph appear to 
lack the depressant, and continued excessive mitosis may occur. Both in the neighbourhood of wounds and in cancerous growth there is a maximum response to cestrone; the two regulators would appear to be in abeyance. These results are discussed in relation to ovarian fupction, pregnancy and cancer.

Yagnetic Field Calculaton by Relaxation Method

Is a pape Motz and W. D. Worthy ( $J$. Inst. Flec. W. (2, Pt. 2, No. 30, December. 1945), of retIf $d$ computing magnetic and electric fields if e) plained with reference to the problem of the mgnetic flux distribution between stator and rotor of dynamo-electric machines. Mathematically, the problem is that of solving Laplace's or Poisson's equation. It is shown how approximate solutions are obtained with the help of a simple arithmetical technique. The differential equations are first replaced by a system of algebraic equations which are then solved by means of the computation method due to R. V. Southwell. The process is outlined and illustrated by a simple example which shows all the essential steps involved. A solution is given for two idealized examples of pole shoe and armature for which rigorous solutions are known. The agreement between the rigorous and approximate solutions is found satisfactory. Sharp corners of pole shoe and armature present a problem for which various solutions are possible, and some of these are indicated. The flux distribution of a complete machine is computed and presented as an example of a complex problem not amenable to a rigorous solution.

\section{High-Frequency Alternators}

A PAPER by Dr. WJ. H. Walker (J. Inst. Elec. Eng., 93 , Pt. 2 No 9 , February 1946) reviews the various types of Herpars available for the generation of frequencid up to $50,000 \mathrm{c} / \mathrm{s}$., and shows that for the nfajority of applications the modern heteropolar indictor alternator is the most suitable machine. tt demonstrates that this altermator is superior to the older homopolar and heteropolar types, owing, in the case of machines required for frequencies of $400-3,600 \mathrm{c} . / \mathrm{s}$. , to a system of field and armature windings which substantially reduces the number of armature conductors required to generate a given electromotive force. For frequencies greater than $3,600 \mathrm{c} . / \mathrm{s}$. this superiority is due to a combination of these windings with a stator-slotting arrangement which permits, for a given frequency and speed of rotation, a large reduction in the number of wound stator slots. The electrical characteristics and mechanical construction of these alternators are discussed, together with their application to the melting of metals and surface-hardening of steel. An appendix gives a brief mathematical treatment of the theory of their novel features.

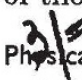

CoNSIDERABLe inte fest has recently been shown in the deterflipg 6 of the physical properties of pure Adddlrbons, and the results of A. I. Vagel (s. Whin. Soc., 133; 1946) on the para hpro and refractivities of the normal hydrocorlon. $\mathrm{fipm} n$-pentane to $n$-hexadecane add some valuable material to this field. The results differ markedly from hitherto accepted figures, and since the atomic and structural parachors and refractivities depend on the values of the $\mathrm{CH}_{2}$ grouping, the calculations based on these will require modification. The mean values for $\mathrm{CH}_{2}$ are : $[P]=$
$39 \cdot 98, R_{C}=4 \cdot 618, R_{D}=4 \cdot 643, R_{F}=4 \cdot 687, R_{G}=$ $4 \cdot 731$ and $M n_{D}^{20}=20 \cdot 63$. Taking into account the values found for $n$-alkyl chlorides, bromides and iodides, the average values $[P]=40.02, R_{C}=4.624$, $R_{D}=4 \cdot 647, R_{F}=4 \cdot 695, R_{G}=4 \cdot 735$, and $M n_{D}^{20}=$ 20.59 are found. It is suggested that the data of Eisenlohr for the refractivities are less accurate than has been supposed.

Structure of Vanadium Tetrackloride

THE strquture of th vanadium tetrachloride $\left(\mathrm{VCl}_{4}\right)$ ifpletwe has con determined by electron diffactid by W Lipscomb and A. G. Whittaker (J) A her. (Pheno (Soc., 67, 2019; 1945). The molecule is a fogla te rahedron, with $\mathrm{V}-\mathrm{Cl}=2 \cdot 03 \pm 0.02 \mathrm{~A}$. and $\mathrm{Cl}-\mathrm{Cl}=3 \cdot 32 \pm 0.03 \mathrm{~A}$. The $\mathrm{V}-\mathrm{Cl}$ distance is in good agreement with that calculated on the assumption that six orbitals are involved in bond formation to the four chlorine atoms and each bond has $\frac{1}{2}$ double bond character. The unpaired electron probably occupies one of the $3 d$ orbitals not involved in bond formation, and it is interesting that this electron does not take any significant steric role (similar to that ordinarily played by an unshared electron pair) in the structure, the observed tetrahedral configuration being that which would be expected if this electron were absent.

Structure of the Globular Star Clusters
E. Finlay-FrEunt. $105,4,237 ; 1945)$ bas attempted to explain the existence of isolated gi filar star clusters in the vicinity of the galaxy. he thain problem presented by the globular sar clutters is their existence as finite systems, isolated from the field of the galactic stars. Their strficture must be determined solely by the gravitational field set up by the stars which constitute such a cluster. But whether the influence of the gravitational field of the whole cluster alone, or in addition, the influence of irregular forces, arising from critical approaches of individual stars, is considered as a decisive factor, it has been shown by Charlier and Martens that the final state should correspond in every case to an isothermal distribution. It has been recently discovered that globular clusters have wide envelopes of faint stars and, taking into consideration these 'atmospheres', the clusters have well-defined finite radii. From these facts the theory is developed that the globular clusters are settling down from an initially adiabatic state, and have reached an intermediate, quasi-stationary state consisting of an isothermal core surrounded by an adiabatic atmosphere. On this view an explanation is afforded of the fact that the most strongly elliptical cores have the lowest atmospheres, and in addition, the fact that the ellipticity, frequently shown in the bright stars, is restricted to the cores, is explained. From the width of the spectral lines in the spectrum of the bright cores, it is possible to determine approximately the masses of globular star clusters, and preliminary estimates show that these masses are very largeof the order $10^{8}$ that of the sun. Although such masses exceed those previously assumed for the masses of the globular clusters, corroborative evidence for the large mass is afforded by the high spatial velocities of the few cluster-type variables discovered outside globular clusters in the galaxy ; and these velocities, it is assumed, can be interpreted as vel. ocities of escape. Such velocities correspond to a mass of about $4 \times 10^{8}$ times the mass of the sun. 